\title{
Re: Reproductive Outcomes of Testicular Versus Ejaculated Sperm for Intracytoplasmic Sperm Injection Among Men with High Levels of DNA Fragmentation in Semen: Systematic Review and Meta- Analysis
}

Esteves SC1, Roque M2, Bradley CK3, Garrido N4

'Androfert, Center for Male Reproduction, Sao Paulo, Brasil

2Origen, Center for Reproductive Medicine, Rio de Janeiro, Brasil

3 Genea, Sydney, New South Wales, Australia

4IVI Foundation, Valencia, Spain

Fertil Steril 2017;108:456-467.e1. doi: 10.1016/j.fertnstert.2017.06.018.

\section{EDITORIAL COMMENT}

Since its introduction in 1992, intracytoplasmic sperm injection (ICSI) has been widely used for different levels of spermatogenic failure in infertile men. However, still reliable methods are not widely accepted for sperm selection for ICSI in male-factor infertility. Sperm DNA plays critical role in embryo development and high sperm DNA fragmentation (SDF) is more common in infertile men compared to fertile individuals (1). In this meta-analysis, the authors aimed to investigate the evidence of potential role of using testicular sperm (testi-ICSI) and ejaculated sperm (ejac-ICSI) for ICSI in nonazoospermic infertile men with confirmed post-testicular SDF. In eligible five studies involving 143 patients, it was found that SDF rate was lower in testicular sperm than in ejaculated sperm. Evaluation of the clinical outcomes of testi-ICSI and ejac-ICSI revealed that, fertilization rates were not different but higher clinical pregnancy and live birth rates were observed in testi-ICSI group whereas miscarriage rates were reduced. In conclusion, in malefactor infertility men with high SDF levels, testicular sperm may have lower SDF compared to ejaculated sperm and couples may benefit from testi-ICSI in terms of better chance of clinical pregnancy and live birth.

\section{Reference}

1. Bungum $M$, Humaidan $P$, Axmon $A$, Spano $M$, Bungum L, Erenpreiss J, Giwercman A. Sperm DNA integrity assessment in prediction of assisted reproduction technology outcome. Hum Reprod 2007;22:174-179.

Emre Bakırcıoğlu, MD

${ }^{\circ}$ Copyright 2017 by the Association of Urological Surgery / Journal of Urological Surgery published by Galenos Publishing House. 